\title{
A study on Security within public transit vehicles
}

\author{
A.N.Seshukumar \\ M. Tech II Year \\ Department of CSE \\ VR Siddhartha Engineering College \\ Vijayawada
}

\author{
Dr.S.Vasavi \\ Professor \\ Department of CSE \\ VR Siddhartha Engineering College \\ Vijayawada
}

\author{
Dr.V.Srinivasa Rao \\ Professor \& HOD \\ Department of CSE \\ VR Siddhartha Engineering College \\ Vijayawada
}

\begin{abstract}
In public transit vehicles, security is the major concern for the passengers. Surveillance Systems provide the security by providing surveillance cameras in the vehicles and a storage that maintains the data. The applications that allow monitoring the data in surveillance systems of public transit vehicles will provide different features to access the video and allow to perform number of operations like exporting video, generating snapshots at a particular time, viewing the live as well as playback videos. This paper studies automation process of video surveillance system that can also be applied in the surveillance system of public transit vehicles. A new feature that enhances the security to the passengers such as tracking of vehicle through the GPS (Global positioning system) tracking system and also capability of providing the vehicle information like acceleration, speed, on the user interface of application to the user.
\end{abstract}

Keywords-Surveillance Cameras; Global Positioning System; Automation; public transit vehicles.

\section{INTRODUCTION}

Surveillance is the monitoring of the behavior, activities, or other changing information, usually of people. It usually refers to observation of individuals or groups by government organizations. The word surveillance may be applied to observation from a distance by means of electronic equipment (such as CCTV cameras), or interception of electronically transmitted information (such as Internet traffic or phone calls). It may also refer to simple, relatively no- or lowtechnology methods such as human intelligence agents and interception. The present surveillance system in public transit vehicles consists of surveillance Cameras mounted within the vehicle and a digital video recorder for the storage of the video data from the cameras. The surveillance system in public transit vehicles will prevent theft by providing onboard security cameras to monitor bus activity and act as preventative measure against acts of theft between riders. The unpredictable

nature of bus passengers throughout the day can many times lead to violent incidents. Such an incident could stem from an argument between riders or a passenger under the influence of alcohol or drugs losing composure. Surveillance cameras can monitor for such unsavory activity, enabling operators to alert authorities. Users of the bus system want to be confident that their mode of transportation is a safe one. Onboard video surveillance cameras give riders the assurance that authorities are doing everything in their power to provide a high level of security. Onboard security cameras can prove valuable in criminal investigations of incidents taking place on buses as well as outside crimes involving specific suspects whose images may be uncovered. Along with all the above specified benefits we propose a new feature that enhances the security is vehicle tracking during the movement of the vehicle in remote location and also providing the acceleration, speed, direction of the vehicle during the motion of it. So, that at any point when ever any unusual events happens the authorities can take immediate decision and also it reminds the driver to drive in controlled manner without crossing the limited field. This tracked information should be displayed on the user interface of the application while playing the live/playback video. We also studied the automation process of video surveillance systems in a static location like shopping malls, airports etc., The main aim of video surveillance is to develop intelligent video surveillance to replace the traditional passive video surveillance that is proving ineffective as the number of cameras exceed the capability of human operators to monitor them. The aim of visual surveillance is not only to put cameras in the place of human eyes, but also to accomplish the entire surveillance task as automatically as possible.

This paper presents related work on this automation process which can also be applicable to surveillance of public transit vehicles. Section 2 provides summary of existing approaches. Our Proposed approach is given in section 3 . Conclusions and future work are given in section 4 and 5 .

\section{RELATED WORK}

This section presents information on Global positioning system and a study on the automation process of video surveillance systems.

The surveillance applications provide features like live/playback video monitoring, exporting the video, generating snapshots. To enhance the security of passengers a new feature namely tracking vehicle while monitoring the video can be added to the existing system. Here we present information on global positioning system.

Global Positioning System is a system that specifies the time and position of an object on the earth. Even though we have different kinds of positioning systems they have their own drawbacks like limited area, some of the positioning systems are LANDMARKS, LORAN and CELESTIAL etc. Since it is satellite based navigation system, it is made up of 27 Earth orbiting satellites among 27 only 24 will be in operation and the remaining 3 are useful when any one among 24 satellites fails. A gps receiver will take the help of 
satellites to find the position of an object on the earth.Here position in the sense location and time of an object. The location is represented by latitude and longitude values based on which location of a vehicle can be identified on the earth.

The process of identifying the suspicious or abnormal behavior in the video from the surveillance cameras requires an operator to identify. If there are hundreds of areas to be monitored, then it needs more number of operators to perform the analysis. Since it requires more number of operators the automation process came into picture. The same can be applicable in case of surveillance in public transit vehicles. We studied the techniques involved in the automation process.

The automation of video surveillance involves the following steps as shown in figure 1.

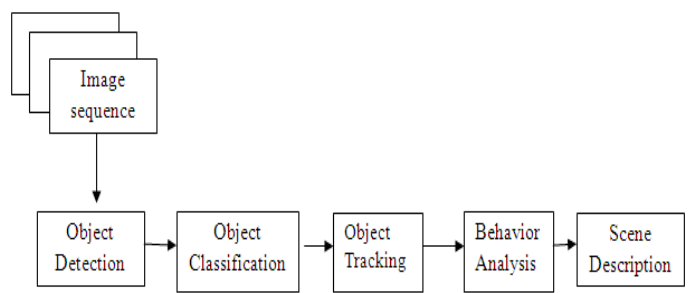

Figure 1: Automation Process of Video Surveillance

The automation process can be done with or without data mining techniques. We studied number of techniques involved in the automation process and the related work on this is presented below.

[1] Presents centralized and decentralized architecture for video surveillance systems. It also presents a typical sequence of video analysis operations in an automatic video surveillance system. It provides about each and every step in automation of video surveillance like preprocessing, Object detection/motion detection, object tracking and object analysis. It explains the algorithms used in motion detection like background subtraction and also that trained object detectors are used to detect objects of a particular category against a complex, possibly moving, background.

[2] Proposed a framework for analysis of surveillance videos by summarizing and mining of the information in the video for learning usual patterns and discovering unusual ones. This framework is useful because it is not possible for a human operator to continuously watch hours of video, either online through a webcam or offline and analyze the video from multiple perspectives. This framework forms the video data in to clusters using an incremental clustering algorithm which can be used with any data type (numerical or symbolic) and is independent of predefining the number of clusters and cluster radii. The incremental clustering algorithm helps in dealing with the large volume of data in case of offline analysis of stored videos. The two techniques component based clustering and cluster algebra for summarization as well as automatic selection of component clusters are used to discover unusual patterns in a surveillance video.

[3] Proposed a review on video surveillance systems. It presents the need of video surveillance and the entire process of video surveillance automation beginning from motion/object detection to behavior analysis. Different techniques are used for the motion segmentation such as background subtraction, temporal differencing and optical flow. Object classification distinguishes between the different objects present in the image into predefined classes such as human, vehicle, animal, clutter, etc. Two approaches namely shape based and motion based classification were used for classification. The final step of an automated surveillance system is to recognize the behavior of the objects and create a high level semantic description of their activities.

[4] Proposed an algorithm for background model initialization. Motion detection and tracking algorithms rely on the process of background subtraction, a technique which detects changes from a model of the background scene. It presents a new algorithm for the purpose of background model initialization. The algorithm takes as input a video sequence in which moving objects are present, and outputs a statistical background model describing the static parts of the scene.

[5] Proposed an approach for automated analysis of passenger's behaviors with a set of visual low-level features, which can be extracted robustly. The approach was performed on a set of global motion features computed in different parts of the image. The complete image, the face and skin color regions, a classification with Support Vector Machines was performed.

[6] Provided a survey on behavior analysis in video surveillance applications. The different methods of behavior analysis were mentioned in the survey. The automation of video surveillance was also provided in detail manner with all the methods in each step of the process.

[7] Proposed Dynamic Oriented Graph method that is used to detect and predict abnormal behaviors, using real-time unsupervised learning. The Dynamic Oriented Graph method processes sequential data from tracked objects, signalizes unusual events and sends alarm warnings for possible abnormal behaviors. This method also constructs a structure to learn and maintain a set of observed patterns of activities, using real-time learning and without the requirement to perform any kind of training. The Dynamic Oriented Graph classifier demonstrated to be extremely fast, learning, classifying and predicting activities on-line and in a dynamical form. The classifier detect the behavior of a very large number of objects in real-time simultaneously.

[8] Proposed an approach for the automatic human behavior recognition and its explanation for video surveillance. This system could automatically report on human activity in video would be extremely useful to surveillance officers who can be overwhelmed with increasingly large volumes of data.

[9] Proposed a framework for moving objects recognition system using its appearance information. Moving objects are extracted with adaptive Gaussian mixture model first. Its silhouette image is unified to a certain mode. Subspace feature of different moving object classes is obtained through training with large numbers of these silhouette images. A more suitable dimension reduction method called marginal Fisher analysis is used to obtain projection eigenvector. 
[10] Proposed a framework for Interactive Motion Analysis for Video Surveillance and Long Term Scene Monitoring. It consists of two feedback mechanisms which allow interactions between tracking and background subtraction. This improves tracking accuracy, particularly in the cases of slow-moving and stopped objects which is completely complement to the existing process.

[11] Proposed a method to detect passengers on-board public transport vehicles with the aim of monitoring their behaviors under suspicious circumstances. It comprises an elliptical head detection algorithm using the curvature profile of the human head as a cue.

[12] Presents real-time implementation of Moving Object Detection Video Surveillance Systems Using FPGA. FPGA is a device that captures the video stream, performs preprocessing, image analysis and reduces the data transfer between FPGA and CPU by transferring the processed results to $\mathrm{CPU}$.

\section{PROPOSED APPROACH}

The present surveillance applications provide the monitoring of live/playback video from the surveillance cameras and allow performing some operations on the video data like exporting video, snapshot generation. But to enhance the security of the passengers we propose a new feature in the surveillance application. The new feature is tracking of vehicle while monitoring the data and also providing the details of the speed, acceleration, direction on the user interface of the application to the user. The tracking of device can be done in two ways.

1. Connecting GPS device separately along with the digital video recorder within the vehicle and receiving the latitude, longitude values from the device and mapping the location on the maps.

2. Enabling a digital video recorder with GPS Connectivity so that a separate device for GPS tracking can be eliminated.

From the two approaches, the second approach would be less expensive than the first approach. The tracking can be done by using the open source tools like Google maps to track the vehicle.

The tracking system allows the user to find the location of the vehicle at any instant of time and also allow the authorities to react to any unusual events like accidents, bus failure situation immediately. This feature should be enabled on the application side so that whenever the operator monitors the video, he can also track the location of the vehicle. This also makes the driver of the bus to drive in a limited speed.

The architecutre for the proposed approach is shown below in figure 2.The architecture explains that the digital video recorder integrated with the GPS connectivity will allow the operator to track the vehicle on the user interface while monitoring the video simultaneously.

The following figure 2 is the architecture for the proposed framework.

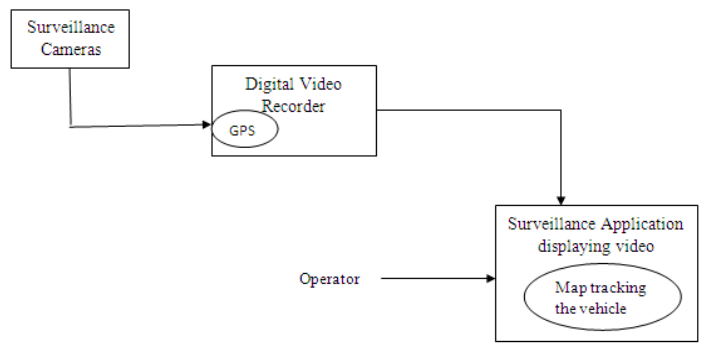

Figure 2: Architecture for enhanced security using GPS tracking system in surveillance applications of public transit vehicles.

The proposed feature can be achieved by using open source Google maps to perform tracking of vehicle. The digital video recorder that contains the gps connectivity will store the latitude and longitude values of the location based on the current position of the vehicle. The application can show the tracking of vehicle by accessing the latitude, longitude values from the device, use the Google maps and shows the exact location of the vehicle. Along with the location, if the device has been provided with the details of the acceleration, speed then the developer can show the details of the acceleration and speed on the application itself.

We also studied the automation process of surveillance systems. We found that the video content from the surveillance cameras contain unstructured enormous data that is not useful for real time processing

[13] Proposed a framework that mines the raw video content from surveillance cameras in surveillance systems of public transit vehicles.

The automation process involves object detection, object classification and object tracking and Behavior analysis. We observed that motion /object detection is being done by enabling it as a feature on digital video recorder itself instead of separate implementation.

Object Tracking gives structure to the observations and enables the object's behavior to be analyzed, for instance detecting when a particular object crosses a line. The aim is to automatically detect passengers which might be a threat to others or themselves.

Object classification is the process of identifying what kind of object is present in the environment and is useful when distinctly different types of objects are present in the environment.

Finally behavior analysis involves analysis and recognition of motion patterns, and the production of high-level description of actions and interactions between or among objects. Human face and gait are now regarded as the main biometric features that can be used for personal identification in visual surveillance systems.

The same process can be applicable to the existing surveillance system of public transit vehicles that would reduce the man power required to monitor the video. 


\section{CONCLUSION}

In this paper, the existing system of surveillance system in public transit vehicles is specified and also proposed a new feature that enhances the security of the passengers. GPS tracking system ensures more security to the passengers. The digital video recorder that is embedded with GPS connectivity can provide the proposed feature. Also the device that is provided with the acceleration and speed details then by accessing those details they can be displayed on the application to the user.

The process of surveillance system needs a human operator to monitor the video data. But a lot of research was done on the automation of surveillance process that reduces the man power required. At present this automation is being done only on applications of static locations. This paper provided the detailed study on the automation process that could be applicable in the surveillance system of public transit vehicles.

\section{FUTURE WORK}

The surveillance applications at present are able to provide the user to monitor the video of a single vehicle at a time in user interface. In future, it can be extended to monitor multiple vehicles simultaneously on a single user interface and also reduce the man power required to monitor the video by implementing the automation process.

The surveillance applications can be provided with the automated process of detecting the violent incidents that may take place in the vehicle.

\section{REFERENCES}

[1] "An Introduction to Automatic Video Surveillance", Andrew W. Senior, Privacy Protection in Video Surveillance, 2009.

[2] Ayesha Choudhary, Santanu Chaudhury and Subhashis Banerjee : A Framework for Analysis of Surveillance Videos in Computer Vision,Graphics\& Image Processing ,2008. Sixrh Indian Conference in December 2008.

[3] Garima Sharma: Video Surveillance System: A Review in IJREAS, Volume 2 Issue2 February 2012.
[4] D. Gutchess†', M. Trajkovi'cł, E. Cohen-Solalł, D. Lyonsł, A. K. Jain†: A Background Model Initialization Algorithm for Video Surveillance in IEEE transactions 2001.

[5] Dejan Arsi'c, Bj"orn Schuller and Gerhard Rigoll:Suspicious Behavior Detection in Public Transportation by Fusion of Low-Level video Descriptors in IEEE transactions, 2007.

[6] TeddyKo:A Survey on Behavior Analyis in Video Surveillance Applications paper published in 2011

[7] Duarte Duque, Henrique Santos and Paulo Cortez : Prediction of Abnormal Behaviors for Intelligent Video Surveillance Systems in IEEE Symposium on Computational Intelligence and Data Mining (CIDM 2007).

[8] Neil Robertson, Ian Reid and Michael Brady: Automatic Human Behavior Recognition and Explanation for CCTV Video Surveillance in security journal,2008.

[9] Zhang Yi, Wang Hancheng: A General Framework of Moving Objects Recognition System.

[10] A.W. Senior, Y. Tian, and M. Lu, "Interactive Motion Analysis for Video Surveillance and Long Term Scene Monitoring", in Proc. ACCV Workshops (1), 2010.

[11] Boon Chong Chee, Mihai Lazarescu and Tele Tan: Detection and Monitoring of Passengers on a Bus by Video Surveillance in IEEE transactions, 2007.

[12] Kryjak, Tomasz and Gorgon, Marek (2011) Real-Time Implementation of Moving Object Detection in Video Surveillance Systems Using FPGA.

[13] JungHwan Oh, Babitha Bandi : Multimedia DataMining Framework for Raw Video Sequences in third International Work on Multimedia DataMining 2002.

\section{AUTHORS PROFILE}

A.N. Seshu Kumar is pursuing Master of Technology with specialization in Computer Science and Engineering in V.R.Siddhartha Engineering College, Vijayawada. His current research interests include video surveillance applications, automation of new applications, quality control where his publications are focused.

Dr.S.Vasavi working as professor in the department of Computer Science and Engineering in V.R.Siddhartha Engineering College,Vijayawada has 16 years of teaching experience.Her areas of research are Semantic interoperability, Data mining and Image processing.

Dr.V.Srinivasa Rao working as professor \& HOD in the department of Computer Science and Engineering in V.R.Siddhartha Engineering College,Vijayawada has 22 years of teaching experience.Her areas of research are Bioinformatics, Image processing. 